\title{
Experiência inicial com o uso de adesivo tissular contendo trombina para tratamento do pseudo-aneurisma femoral
}

\author{
Treatment of femoral pseudoaneurysm with thrombin tissue adhesive: initial experience \\ D aniel Mendes Pinto ${ }^{1}$, José 0 limpio D ias Júnior ${ }^{2}$, Bernardo Lopes $C$ ançado Fonseca³, \\ Rodrigo D aniel M oreialvar' ${ }^{1}$, Leonardo G hizoni Bez ${ }^{1}$, C aetano de Sousa Lopes ${ }^{1}$
}

\begin{abstract}
Resumo
0 pseudo-aneurisma (PSA) após cateterização femoral tem sido diagnosticado com regularidade em serviços com grande movimento de intervenções percutâneas, com incidência variando de 0,05 a 6\%. PSA femorais pequenos podem ser acompanhados até a resolução espontânea. As opções de tratamento são: compressão guiada por ultrasom, injeção de trombina para trombose do PSA e tratamento cirúrgico. A injeção percutânea de trombina tem a vantagem de ser um procedimento indolor e rápido. Podem ser utilizados trombina isolada ou preparados contendo trombina associada a fibrinogênio e fatores de coagulação. A experiência inicial dos autores de cinco casos tratados com injeção de adesivo tissular contendo trombina mostrou resultado satisfatório em quatro; um caso necessitou tratamento cirúrgico. $\mathrm{N}$ ão houve sucesso com uso isolado de trombina humana, porém, ocorreu trombose imediata após injeção de preparado de trombina associada a fibrinogênio/fator XIII. N este artigo, são discutidas as opções de tratamento dos PSA femorais e a técnica do uso de trombina percutânea.
\end{abstract}

Palavras-chave: Pseudo-aneurisma, ultra-som, trombina, fibrinogênio.

\section{Introdução}

0 pseudo-aneurisma(PSA) femoral ocorreem 0,05 a $6 \%$ das vezes após punção para cateterismo da artéria femoral ${ }^{1,2}$. Alguns fatores de risco estão associados ao seu desenvolvimento, como o calibre do sistema de

1. Cirurgiões vasculares, Especialistas em Angiologia e Cirurgia Vascular, Sociedade Brasileira de Angiologia e de Cirurgia Vascular.

2. Cardiologista, Especialista em Ecografia Vascular e Ecocardiografia, Sociedade Brasileira de Cardiologia.

3. Radiologista, Membro Titular, Colégio Brasileiro de Radiologia.

Este estudo foi realizado nos hospitais Mater Dei, Felício Rocho e Lifecenter, Belo Horizonte, MG.

Artigo submetido em 13.02.06, aceito em 16.03.06.

$J$ Vasc $\mathrm{Br} 2006 ; 5(1): 30-6$

Copyright $\odot 2006$ by Sociedade Brasileira de Angiologia e de Cirurgia Vascular.

\begin{abstract}
Pseudoaneurysms caused by femoral artery catheterization have been regularly diagnosed in medical units with a great number of percutaneous interventions, with a documented incidence between 0.05 and $6 \%$. Small femoral pseudoaneurysms undergo spontaneous resolution. Treatment options are: ultrasound-guided compression, thrombin injection to inducepseudoaneurysm thrombosis and surgical treatment. Percutaneous thrombin injection has the advantage of being a fast and painless procedure. Both isolated thrombin and thrombin preparations with fibrinogen and coagulation factors can be used. The authors' initial experience with five cases treated with thrombin tissue adhesive showed successful results in four; one case required surgery. There was no success with isolated human thrombin, but immediate thrombosis was achieved after injection of thrombin associated to fibrinogen and factor XIII. In this article, the treatment options for femoral pseudoaneurysms and the technique of percutaneousthrombin are discussed.
\end{abstract}

Key words: Pseudoaneurysm, ultrasonography, thrombin, fibrinogen.

punção arterial e o uso de anticoagulantes ${ }^{3}$. As opções para o tratamento do PSA são: tratamento observador (resolução espontânea do PSA), compressão guiada por ultra-som (CGU), injeção de trombina guiada por ultra-som (IT GU), tratamento endovascular e cirurgia convencional.

Cope $\&$ Z eith 4 publicaram em 1986 os primeiros casos de IT GU para tratamento de PSA. Várias publicações relatam o tratamento percutâneo com injeção de trombina como de escolha para os PSA femorais, com taxas de sucesso em torno de $97 \%$ e com $2 \%$ de complicações ${ }^{5-7}$. Apesar das séries com estudos prospectivos apresentarem um número ainda reduzido de pacientes, existem vantagens sobre a simples CGU, 
como maior comodidade para o pacientee para o ultrasonografista, rápida resolução dos sintomas (principalmente da dor em região inguinal) e menor taxa de reincidência ${ }^{8}$. As desvantagens incluem 0 custo do procedimento eo uso dematerial heterólogo - trombina ou aprotinina bovina - , que pode induzir a reações antigênicas ${ }^{9}$.

O sobjetivos destetrabal ho são relatar a experiência inicial de cinco casos de PSA tratados com injeção percutânea de trombina isolada e associada a adesivos tissulares, descrever a técnica usada pelos autores e discutir sobre as opções atuais de tratamento.

\section{Pacientes e técnica}

Foi realizado tratamento de cinco pacientes, três homens eduas mulheres, idade de 52 a 82 anos (média de 67 anos), com PSA femorais, todos em região inguinal direita, da artéria femoral comum, ocorridos após punção femoral para procedimentos arteriográficos cardíacos ou cerebrais. Foram selecionados para tratamento com adesivo tissular os casos de PSA que não seresolveram com aC GU ( 2 casos) eaquel es muito sintomáticos, nos quais julgou-se que a compressão isolada seria muito dolorosa. Foram excluídos os pacientescom PSA pequenose de resolução fácil com a compressão isolada, além daquel es com indicação para tratamento cirúrgico precoce, especialmente casos de instabilidade hemodinâmica devido a sangramento. Em todos os casos, foi realizado consentimento informado sobre o procedimento.
0 preparado detrombina associado a adesivo tissular utilizado foi o Beriplast ${ }^{\circledR}$ (Aventis Behring), que consisteem dois componentesquepodem ser injetados simultaneamente: trombina humana (concentração de 400 a 600 UI/ml), reconstituída em solução de cloreto de cálcio (preparado 1); e fibrinogênio humano e fator XIII, reconstituídos em solução de aprotinina bovina (preparado 2). Os dois preparados, quando injetados simultaneamente, formam um adesivo elástico, usado para hemostasia em superfícies cirúrgicas cruentas ou áreas de anastomose vascular.

\section{Técnica da ITG U}

O s procedimentos foram realizados no setor de ecografia vascular de hospitais em Belo H orizonte, no período de maio a outubro de 2005. Foram feitos infiltração anestésica em local com lidocaína 1\% em todos os pacientes e preparo da região inguinal com campos cirúrgicos estéreis.

O s dois componentes do preparado de trombina são reconstituídos conforme orientação do fabricante. 0 conteúdo do frasco de trombina liofilizada é transferido, por vácuo, para o solvente (solução de cloreto de cálcio), resultando em solução com 400 a $600 \mathrm{UI}$ de trombina/ml. 0 conteúdo do frasco de fibrinogênio efator XIII liofilizadostambém étransferido, por vácuo, para o frasco com solvente (solução de aprotinina bovina). Após 2 minutos em repouso, as soluções são aspiradas, cada uma em uma seringa (Figura 1).

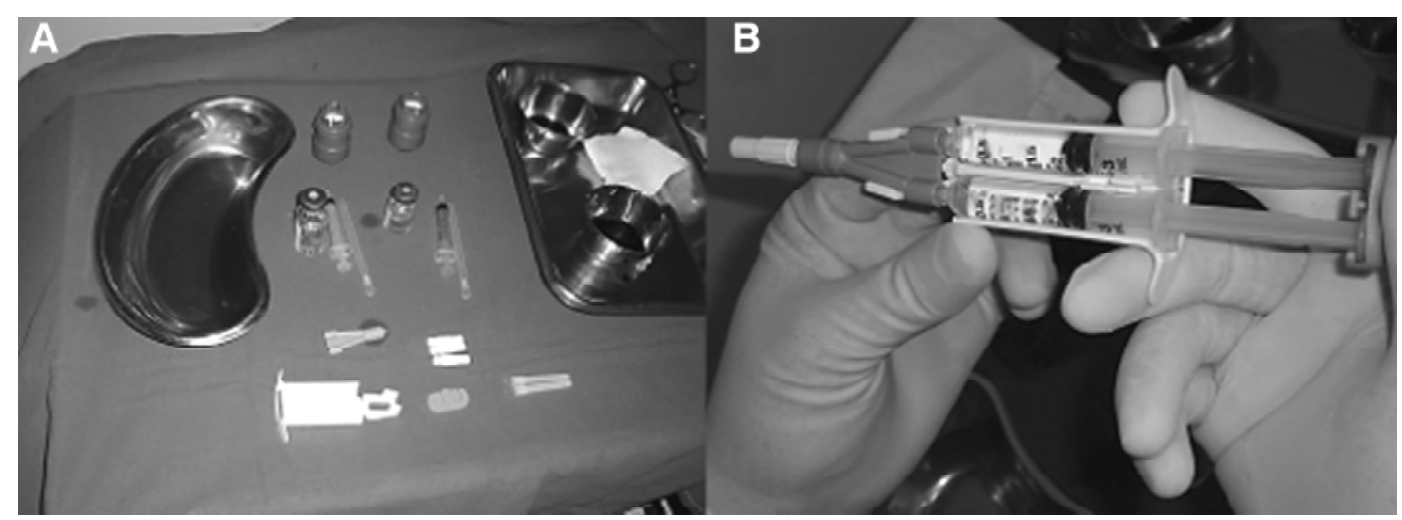

Figura 1 - Kit com preparados de trombina e fibrinogênio/fator XIII e material para punção (A); seringa contendo preparado de trombina, e outra com fibrinogênio/fator XIII para injeção simultânea (B) 
Realizamosa injeção do preparado deadesivo tissular associada à compressão do colo do PSA com o transdutor do ultra-som. 0 método consiste em levar à trombose do PSA, enquanto o fluxo é interrompido pela compressão do colo pelo transdutor de ultra-som, mantendo o fluxo pela artéria femoral. Guiada por ultra-som, uma agulha $20 \mathrm{G}$ ou $22 \mathrm{G}$ éposicionada no interior do PSA. Algumas vezes, injetamos solução sal ina para melhor visualização da agul ha. Pode ser feita injeção simultânea de trombina e fibrinogênio/fator XIII ou isolada de trombina. D urante a injeção, é feita compressão do colo do PSA com o transdutor do ultrasom. A compressão do colo é mantida por aproximadamente 2 minutos. A trombose pode ser visualizada em poucos segundos, e nota-se trombo hiperecogênico no interior do saco aneurismático (Figura 2).

É feito curativo compressivo, e o paciente é orientado a não movimentar o membro inferior por 12 horas. Após esse período, usualmente no dia seguinte ao do procedimento, o curativo é retirado, e o paciente é liberado para deambulação.

\section{Resultados}

O diagnóstico do PSA foi feito por ultra-sonografia realizada após suspeita clínica, em um intervalo de até 7 dias após os procedimentos.

0 maior diâmetro dosPSA variou de 4,5 a 8,0 cm. Em quatro casos, OSPSA eram multilobulados. Em um caso, foi realizado tratamento percutâneo de persistênciado canal atrial em paciente de 52 anos. T ratava-se de PSA volumoso, de 8,0 × 4,2 cm de diâmetro, multilobulado, no qual era difícil a visualização do colo.
D oscinco casosrealizados, houvetrombose do PSA em quatro, todos com injeção de trombina associada a fibrinogênio/fator XIII (T abela 1).

Em dois casos, foi usada inicialmente trombina humana isolada. Em um dos casos, não conseguimos a trombose do PSA após injeção de aproximadamente 500 U I (1 ml) de trombina; realizamos, então, injeção de mais $500 \mathrm{UI}$ trombina $(1 \mathrm{ml})$ associada a fibrinogênio/fator XIII ( $1 \mathrm{ml}$ ), com trombose imediata do PSA.

$\mathrm{N}$ o outro caso, usamos inicialmente $500 \mathrm{UI}$ de trombina ( $1 \mathrm{ml}$ ). Visualizamos trombos no interior do saco aneurismático, composto por vários lóbulos (multiloculado). Após 10 minutos de compressão, não houve trombose dos outros lóbulos. Injetamos, então, mais $500 \mathrm{UI}$ de trombina ( $1 \mathrm{ml}$ ) associada a fibrinogênio/fator XIII (1 ml), com trombose imediata total de todo o PSA.

Em outros dois casos, injetamos trombina associadaafibrinogênio/fator XIII, com tromboseimediatado PSA, conforme descrito na T abela 1.

Em um caso, tentamos a injeção de trombina associada a fibrinogênio/fator XIII, porém, não houve sucesso. T ratava-se dePSA degrandetamanho (aproximadamente $6 \mathrm{~cm}$ de diâmetro), em paciente do sexo feminino e de baixa estatura, que foi submetida a tratamento percutâneo de fechamento de canal arterial com endoprótese, através deintrodutor 12 Fr. 0 acesso para essa intervenção éfeito pela veia femoral, eocorreu lesão iatrogênica da artéria femoral; apesar disso, não apresentava fístula arteriovenosa. Após tentativa de compressão por ultra-som sem sucesso, essa pacientefoi submetida a tratamento cirúrgico do PSA.

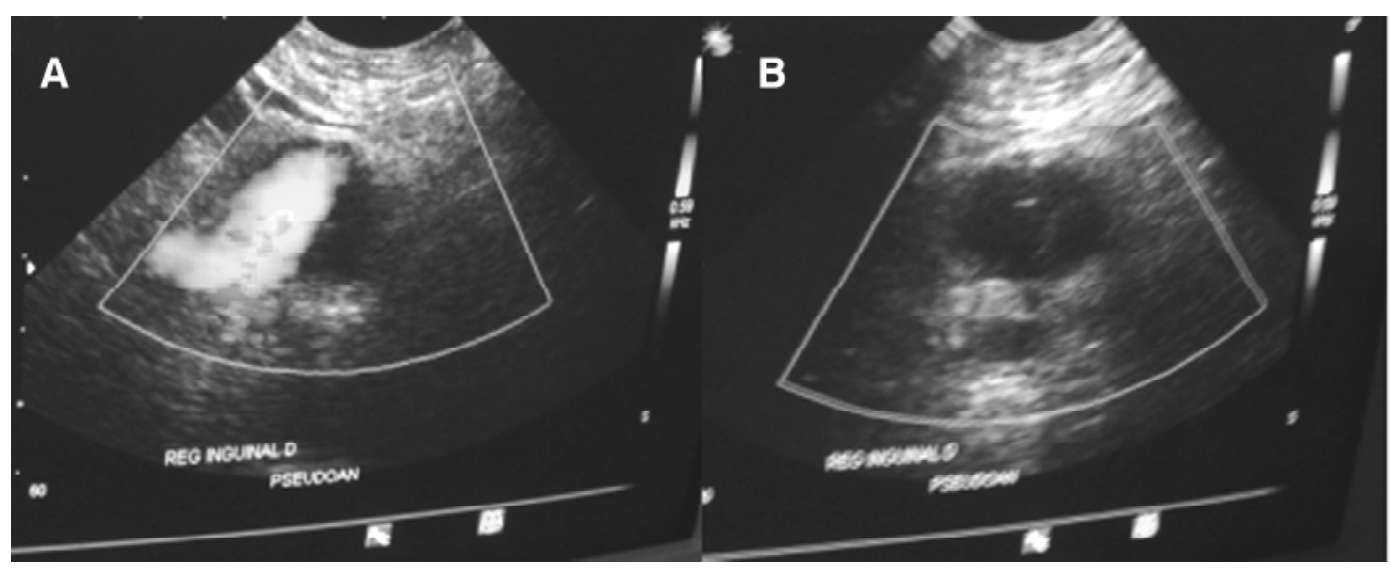

Figura 2 - PSA em região inguinal direita (A); PSA trombosado (B) 
T abela 1 - Características dos pacientes submetidos a tratamento do PSA femoral

\begin{tabular}{|c|c|c|c|c|c|c|}
\hline Caso & $\begin{array}{r}\text { Causa } \\
\text { do PSA }\end{array}$ & $\begin{array}{l}\text { T entativa } \\
\text { prévia de } \\
\text { compressão } \\
\text { guiada por } \\
\text { ultra-som }\end{array}$ & $\begin{array}{l}\text { Trombina } \\
\text { isolada }\end{array}$ & $\begin{array}{l}\text { Trombina } \\
\text { associada a } \\
\text { fibrinogênio/ } \\
\text { fator XIII }\end{array}$ & $\begin{array}{l}\text { D ose total } \\
\text { de trombina } \\
\text { utilizada }\end{array}$ & Resultado \\
\hline 1 & $\begin{array}{l}\text { Angioplastia } \\
\text { coronária, } \\
\text { introdutor } 5 \mathrm{Fr}\end{array}$ & N ão & - & $\begin{array}{c}0,6 \mathrm{ml} \text { trombina } \\
+0,6 \mathrm{ml} \\
\text { fibrinogênio/ } \\
\text { FXIII }\end{array}$ & 480 a 720 UI & $\begin{array}{c}\text { Trombose } \\
\text { do PSA }\end{array}$ \\
\hline 2 & $\begin{array}{l}\text { Arteriografia } \\
\text { cerebral, } \\
\text { introdutor } 5 \mathrm{Fr}\end{array}$ & Sim & - & $\begin{array}{c}\text { 1,0 ml de trombina } \\
+1,0 \mathrm{ml} \\
\text { fibrinogênio/ } \\
\text { FXIII }\end{array}$ & 400 a $600 \mathrm{UI}$ & $\begin{array}{c}\text { Trombose } \\
\text { do PSA }\end{array}$ \\
\hline 3 & $\begin{array}{l}\text { Coronariografia } \\
\text { diagnóstica, } \\
\text { introdutor } 5 \mathrm{Fr}\end{array}$ & N ão & $1,0 \mathrm{ml}$ & $\begin{array}{c}\text { 1,0 ml de trombina } \\
+1,0 \mathrm{ml} \text { fibrinogênio/ } \\
\text { FXIII }\end{array}$ & $\begin{array}{c}800 \mathrm{a} \\
1.200 \mathrm{UI}\end{array}$ & $\begin{array}{c}\text { Trombose } \\
\text { do PSA }\end{array}$ \\
\hline 4 & $\begin{array}{c}\text { Fechamento } \\
\text { percutâneo de } \\
\text { canal arterial, } \\
\text { introdutor } 12 \mathrm{Fr}\end{array}$ & N ão & - & $\begin{array}{c}\text { 4,0 ml de trombina } \\
+2,0 \mathrm{ml} \text { fibrinogênio/ } \\
\text { FXIII }\end{array}$ & $\begin{array}{c}1.600 \mathrm{a} \\
2.400 \mathrm{UI}\end{array}$ & Insucesso \\
\hline 5 & $\begin{array}{l}\text { Coronariografia } \\
\text { diagnóstica, } \\
\text { introdutor } 6 \mathrm{Fr}\end{array}$ & Sim & $1,0 \mathrm{ml}$ & $\begin{array}{c}\text { 1,0 ml de trombina } \\
+1,0 \mathrm{ml} \text { fibrinogênio/ } \\
\text { FXIII }\end{array}$ & $\begin{array}{c}800 \mathrm{a} \\
1.200 \mathrm{UI}\end{array}$ & $\begin{array}{c}\text { Trombose } \\
\text { do PSA }\end{array}$ \\
\hline
\end{tabular}

FXIII = fator XIII; PSA = pseudo-aneurisma femoral.

Em dois casos, foi tentada inicialmente a CGU, sem injeção de trombina. N os outros três casos, optamospelainjeção detrombina como primeiro tratamento. Com exceção da pacientequenecessitou decirurgia, os outros permaneceram internados até 0 dia seguinte ao do procedimento.

$\mathrm{N}$ os quatro casos com trombose do PSA, foi feito eco-D oppler vascular de controle num período de 7 a 15 dias após o procedimento. Em todosos casos, o PSA estavatrombosado, eospacientes apresentavam melhora dos sintomas compressivos. $\mathrm{N}$ ão houve complicações relacionadas ao procedimento.

\section{D iscussão}

O PSA femoral éa complicação mais comum após a cateterização femoral. Knight et al. ${ }^{8}$, numa análise retrospectiva de 15.769 pacientes, encontraram inci- dência de 0,28\% dePSA femorais. Incidências maiores são encontradas quando os pacientes são rastreados com eco-D oppler de rotina, levando a diagnóstico de pequenos PSA assintomáticos em 8 a $14 \%{ }^{9}$. A pesar da incidência de PSA femorais ser pequena, o grande volume de procedimentos com cateterização femoral faz com que esse problema esteja sempre presente em serviços de grande movimento. 0 utros fatores contribuem para a incidência regular dos PSA, como o uso cadavez maisrotineiro debainhas degrosso cal ibrepara procedimentos percutâneos e de drogas antiplaquetárias em associação, como os inibidores do complexo llb/ IIl a associados a clopidogrel e aspirina ${ }^{10}$.

São fatores de risco descritos na literatura para a formação dos PSA: sexo feminino, intervenção percutânea terapêutica, tamanho do cateter, anticoagulação terapêutica, uso de aspirina e outra medicação antipla- 
quetária associada e não-utilização de mecanismo de fechamento arterial percutâneo $2,8,11$.

M uitosPSA não necessitam de tratamento. Q uando pequenos, usualmente com diâmetro menor que 2 $\mathrm{cm}$, podem trombosar espontaneamente, sem necessitar compressão ${ }^{2}$. 0 tratamento cirúrgico era a opção habitual até 1991 , quan do F el meth et al. ${ }^{12}$ descreveram a técnica de CGU. A CGU tem se mostrado eficaz; entretanto, a compressão é dolorosa e demorada e apresenta maior fal ha terapêutica em pacientes anticoagulados ou com PSA maiores 3,13 . 0 tempo de compressão pode variar de 10 a 300 minutos, com taxa de sucesso de $75 \%{ }^{6}$. Alguns pacientes não toleram 0 procedimento por causa da dor, e há séries mostrando necessidade de sedação anestésica em até $42 \%$ dos casos $^{14}$. Brophy et al. ${ }^{15}$ mostraram que as taxas de falência podem aumentar para 38 a 48\% quando são incluídos na anál ise estatística pacientes que interromperam a compressão por motivos técnicos ou devido à dor. Além disso, a CGU não é isenta de complicações. São descritas embolização distal, trombose da veia femoral comum, trombose da artéria femoral comum e bradicardia durante a compressão ${ }^{16}$.

A ITGU para tratamento dos PSA foi descrita inicialmentepor Cope\& Z eith ${ }^{4}$ em 1986; descreveram quatro pacientes com PSA femoral, ilíaco eem artérias tibiais. Entretanto, foram Kang et al. que descreveram a técnica utilizada hoje, com 95\% de sucesso em 21 casos $^{17}$. Em 2000, esses autores publicaram uma série com 74 casos com $96 \%$ de sucesso ${ }^{6}$. Várias publicações mostram taxas de sucesso acima de 95\%11,18,19. Além do alto índice de trombose do PSA, outras vantagens importantes são a facilidade do procedimento percutâneo e sua rapidez, com melhor tolerância do paciente, levando alguns autores a indicar o procedimento como preferencial sobre a CGU (T abela 2) ${ }^{20}$.

$\mathrm{N}$ a técnica original descrita por Kang et al., nenhuma compressão éfeita durantea injeção detrombina ${ }^{17}$.

T abela 2 - V antagens da IT GU

Sem necessidade de sedação anestésica

Elevado sucesso técnico

M elhor tolerância pelo paciente

M enor tempo de internação hospitalar (comparado ao tratamento cirúrgico)
Loose \& H aslam descreveram a preocupação com a embolização distal devido ao fluxo circular no PSA, associada à reversão do mesmo para a artéria femoral durante a diástole ${ }^{21}$. Esses autores propuseram a oclusão do colo por via endovascular, com cateter-balão. O s estudos que relatam a injeção de trombina associada à oclusão do colo com balão são poucos e com poucos $\operatorname{casos}^{22}$. A associação da técnica endovascular torna 0 procedimento mais trabal hoso e caro, e não há evidência de benefício, ou seja, demenor chance de embolização distal da trombina². Em várias séries de casos, não há relato sehá ou não compressão do colo pelo transdutor do ultra-som ${ }^{5,11}$. Foi com a preocupação teórica de evitar a embolização que nós real izamos a compressão do colo do PSA, com o transdutor, durantea injeção do adesivo tissular.

A trombose do PSA após injeção de trombina é rápida evisualizada pela ausência defluxo à análisecom D oppler colorido. D anzi et al. relataram que a trombose ocorreu após $12 \pm 15$ segundos após a injeção ${ }^{14}$. Poucos são os estudos que descrevem a injeção associada de fibrinogênio/fator XIII21,22. A associação de fibrina, fibrinogênio, fator XIII e cálcio é conhecida como adesivo tissular e utilizada como hemostático tópico em áreas de sutura. D oses variadas de trombina são necessárias para induzir a trombose do PSA, sendo descritas de 100 UI a 6.000 UI; porém, a maioria dos PSA necessita de doses entre 500 e 1.000 U $^{11,14}$.

Em dois casos, usamos inicialmente trombina isolada; porém, não houve trombose do PSA após compressão de aproximadamente 10 minutos. $N$ estes casos, a trombose do PSA ocorreu poucos segundos após a injeção de trombina associada a fibrinogênio/fator XIII. N otamos que o trombo, após a injeção de trombina e fibrinogênio/fator XIII, é mais hiperecogênico e mais facilmente visualizado do que o trombo após injeção isolada de trombina, fato descrito por outros autores ${ }^{9,21,22}$. N ão sabemos explicar por que não houve sucesso com a injeção isolada de trombina, e o número reduzido de casos não permite levar a conclusões sobrea maior eficácia do adesivo tissular. $\mathrm{N}$ ão há publicações comparando a injeção isolada de trombina com o uso associado de fibrinogênio ou outros fatores de coagulação.

Publicações mais recentes indicam uso de doses reduzidas de trombina humana, podendo chegar a doses baixas, como 100 U I11. Porém, o uso da trombina em pequenas doses emaiores volumes écontroverso, e há autores que preferem usar doses maiores em volu- 
mes menores. A tendência atual é o uso de doses menores de trombina, com injeção de intervalos de $100 \mathrm{UI} / \mathrm{ml}$, até que ocorra a trombose ${ }^{18,19}$.

Para PSA grandes e com sinais de complicação, como isquemia distal, infecção e compressão nervosa, 0 tratamento cirúrgico é a melhor escolha (T abela 3). 0 insucesso que tivemos no caso 4 foi tratado com cirurgia. N ão conseguimos a visualização do colo do PSA devido ao hematoma volumoso. Além disso, foi uma lesão da artéria femoral com introdutor $12 \mathrm{Fr}$. Acreditamos que essas foram as causas de não conseguirmos a trombose com a compressão e injeção de trombina.

Tabela 3 - Contra-indicações ao tratamento percutâneo do PSA femoral

PSA em rápida expansão

PSA infectado

Isquemia distal

D or neuropática (necessita descompressão)

$\mathrm{N}$ ecrose de pele

Critérios ultra-sonográficos:

D iâmetro maior que $8 \mathrm{~cm}$ associado ànão-visualização do colo do PSA.

PSA = pseudo-aneurisma.

São descritos métodos endovasculares para tratamento dos PSA. A injeção de trombina pode ser feita com cateter intra-arterial através do colo do PSA ${ }^{23}$. Entretanto, ainjeção intra-arterial não éusada paraPSA femorais e é indicada para locais onde não há possibilidade de punção percutânea ${ }^{24}$.

À ITGU, pode-se associar o uso de balão intraarterial para proteção da artéria femoral. Q uando o colo é largo e curto, um cateter-balão é colocado na artéria femoral através de acesso femoral contralateral, com o objetivo de prevenir a embolização. Entretanto, essa técnica não se mostrou superior à simples compressão do colo pelo transdutor do ultra-som²1,25.

Aytekin et al., em publicação recente, relataram 0 uso de n-butil cianoacrilato (N BCA) percutâneo para trombose do PSA femoral em 18 pacientes $^{25}$. U sando a diluição 50:50 com Lipiodol, ocorre rápida polimerização, com trombose do PSA. Trata-se de técnica interessante, especialmente devido aos custos menores do N BCA, que pode ser usado em hospitais onde não há disponibilidade de trombina humana.

A principal complicação da injeção percutânea de trombina é a embolização distal devido ao escape de material para artéria femoral. Para prevenir a embolização, deve ser feita compressão do colo do PSA com o transdutor de ultra-som durante a injeção. Complicações como trombose venosa e abscesso inguinal são pouco comuns ${ }^{26,27}$. H á poucos casos de embolia para artéria femoral descritos queforam tratados com cirurgia ou trombolíticos intra-arterial ${ }^{28,29}$.

$\mathrm{N}$ a nossa pequena série, o uso associado de fibrinogênio/fator XIII mostrou resultados melhores, com trombose imediata do aneurisma. $\mathrm{N}$ ão conseguimos trombose do PSA com injeção isolada de trombina. U ma desvantagem da associação com fibrinogênio/ fator XIII éa necessidade de reconstituição em solução de aprotinina de origem bovina. Apesar de a trombina utilizada ser de origem humana, o uso da aprotinina bovina pode levar a reações al érgicas, numa nova exposição futura ${ }^{30}$. Há casos que descrevem desde reações discretas até grave anafilaxia após nova injeção de produtos de origem bovina ${ }^{31}$.

Concluindo, o tratamento do PSA femoral com injeção de trombina apresenta uma série de vantagens, como a simplicidade, rápida resolução e baixa taxa de complicações. 0 custo do procedimento ainda é um fator limitador em nosso meio. PSA femorais acima de $3 \mathrm{~cm}$, que não fecharam após a CGU, ou multiloculados são tratados de maneira eficaz com a injeção de trombina. Em nosso número reduzido de casos, houve melhor resposta com a associação de fibrinogênio/fator XIII.

\section{R eferências}

1. Lumsdem AB, M iller JM, Kosinski $A S$, et al. A prospective evaluation of surgically treated groin complications following percutaneous cardiac procedures. Am Surg. 1994;60:132-7.

2. Morgan R, Belli $A M$. Current treatment methods for postcatheterization pseudoaneurysms. J V asc Interv Radiol. 2003;14:697-710.

3. H ye RJ. Compression therapy for acute iatrogenic femoral pseudoaneurysms. Semin V asc Surg. 2000;13:58-61.

4. Cope C, Zeit R. Coagulation of aneurysms by direct percutaneous thrombin injection. AJR Am J Roentgenol. 1986;147:383-7.

5. Tamin WZ, Arbid EJ, Andrews LS, Arous EJ. Percutaneous induced thrombosis of iatrogenic femoral pseudoaneurysms following catheterization. Ann Vasc Surg. 2000;14:254-9. 
6. Kang SS, Lapropoulos N, M ansour M A, et al. Expanded indications for ultrasound-guided thrombin injection of pseudoaneurysms. J V asc Surg. 2000;31:289-98.

7. Gale SS, Scissons RP, Jones L, Salles-Cunha SX. Femoral pseudoaneurysm thrombinjection. Am J Surg. 2001;181: 379-83.

8. Knight CG, Healy DA, Thomas RL. Femoral artery pseudoaneurysms: risk factors, prevalence and treatment options. Ann Vasc Surg. 2003;17:503-8.

9. Middleton WD, D asyam A, T eefey SA. Diagnosis and treatment of iatrogenic femoral artery pseudoaneurysms. U Itrasound Q . 2005;21:3-17.

10. Demirbas O, Batyraliev T, Eksi Z, Pershukov I. Femoral pseudoaneurysm due to diagnostic or interventional angiographic procedures. Angiology. 2005;56:553-6.

11. O Isen DM, Rodriguez JA, V ranic M, Ramaiah V, Ravi $R$, $D$ iethrich EB. A prospective study of ultrasound scan-guided thrombin injection of femoral pseudoaneurysm: atrend toward minimal medication. J V asc Surg. 2002;36:779-82.

12. Fellmeth BD, RobertsAC, Bookstein J, et al. Postangiographic femoral artery injuries: non-surgical repair with ultrasound guided compression. Radiology. 1991;178:671-5.

13. Lonn L, Olmarker A, G eterud K, Risberg B. Prospective randomized study comparing ultrasound-guided thrombin injection to compression in the treatment of femoral pseudoaneurysms. J Endovasc Ther. 2004;11:570-6.

14. D anzi G B, Sesana M , C apuano C , et al. Compression repair versus low-dose thrombin injection for the treatment of iatrogenic femoral pseudoaneurysm: a retrospective casecontrol study. Ital H eart J. 2005;6:384-9.

15. Brophy D P, Sheiman RG, AmatulleP, A kbari CM . I atrogenic femoral pseudoaneurysms: thrombin injection after failed U S-guided compression. Radiology. 2000; 214:278-82.

16. Taylor BS, Rhee RY, M uluk $S$, et al. Thrombin injection versuscompression of femoral artery pseudoaneurysms. J V asc Surg. 1999;30:1052-9.

17. Kang SS, Labropoulos N, M ansour MA, Baker WH. Percutaneous ultrasound guided thrombin injection: a new method for treating postcatheterization femoral pseudoaneurysms. J V asc Surg. 1998;27:1032-8.

18. Kruger $K$, Zaehringer $M$, Sohngen FD, et al. Femoral pseudoaneurysms: management with percutaneous thrombin injections - success rates and effects on systemic coagulation. Radiology. 2003;226:452-8.

19. K rueger K, Zaehringer M , Strohe D, Stuetzer $H$, Boecher J, Lackner K. Postcatheterization pseudoaneurysms: results of U S-guided percutaneous thrombin injection in 240 patients. Radiology. 2005;236:1104-10.

20. F riedman SG, Pellerito JS, Scher L, Faust G, BurkeB, Safa T . U Itrasound-guided thrombin injection is the treatment of choice for femoral pseudoaneurysms. Arch Surg. 2002;137: 462-4.
21. LooseH W, H aslam PJ. Themanagement of peripheral arterial aneurysms using percutaneous injection of fibrin adhesive. $\mathrm{Br}$ J Radiol. 1998;71:1255-9.

22. M atson M B, M organ RA, Belli AM . Percutaneous treatment of pseudoaneurysms using fibrin adhesive. $\mathrm{Br} J$ Radiol. 2001;74:690-4.

23. W alker T G, G eller SC, Brewster D C. T ranscatheter occlusion of a profundafemoral artery pseu doaneurysm using thrombin. AJR Am J Roentgenol. 1987;149:185-6.

24. EngelkeC, Q uarmby J, U bhayakar G, M organ R, H olmesK, Belli AM . Autologousthrombin: anew embolization treatment for traumatic intrasplenic pseudoaneurysm. J Endovasc Ther. 2002;9:29-35.

25. Aytekin C, Firat A, Yildirim E, K irbasI , Boyvat F. U Itrasoundguided glue injection as alternative treatment of femoral pseudoaneurysms. Cardiovasc Intervent Radiol. 2004;27: 612-5.

26. Kurz DJ, Jungius KP, Luscher TF. Delayed femoral vein thrombosis after ultrasound-guided thrombin injection of a postcatheterization pseudoaneurysm. J V asc Interv Radiol. 2003;14:1067-70.

27. Paulson EK, N elson RC, M ayes CE, Sheafor DH, Sketch M H $\mathrm{J} r$, K liewer M A. Sonographically guided thrombin injection of iatrogenic femoral pseudoaneurysms: further experience of a singleinstitution. AJR Am J Roentgenol. 2001;177:309-16.

28. Sackett WR, T aylor SM, C offey CB, et al. U Itrasound-guided thrombin injection of iatrogenic femoral pseudoaneurysms: a prospective analysis. Am Surg. 2000;66:937-40.

29. ZargeJ, VIlimureP, M athewson C, Lawrence J. Complications related to thrombin injection for pseudoaneurysm repair. J $V$ asc T ech. 2001;25:209-12.

30. Vazquez $V$, Reus $M$, Pinero $A$, et al. Human thrombin for treatment of pseudoaneurysms: comparison of bovine and human thrombin sonogram-guided injection. AJR Am J Roentgenol. 2005;184:1665-71.

31. PopeM, Johnston KW . Anaphylaxis after thrombin injection of a femoral pseudoaneurysm: recommendations for prevention. J V asc Surg. 2000;32:190-1.

Correspondência:

$D$ aniel $M$ endes Pinto

Av. do Contorno, 9495/01, Prado

CEP 30110-130 - Belo H orizonte, M G

E-mail: dmpnet@terra.com.br 